\title{
Comsol 2D Simulation of Heavy Oil and Bitumen Recovery by SAGD Bo ZHAO ${ }^{1, a,{ }^{*},}$, Yang LIU², Yan-Yan LUO ${ }^{3}$, Guang-Qing ZHANG ${ }^{4}$ \\ ${ }^{1,4}$ Beijing City, Changping District FuXue Road No.18, China University of Petroleum (Beijing) \\ ${ }^{2,3}$ Beijing City, Haidian District Xueyuan Road No.20, CNPC Research Institute of Science and Technology \\ a18810905693@163.com \\ ${ }^{*}$ Corresponding author
}

Keywords: SAGD, Comsol, Framework, Multiphase Coupling.

\begin{abstract}
In western Canada, the heavy oil thermal recovery technology SAGD (steam-assisted gravity drainage) has been widely used in heavy oil recovery, and achieved good production effect. In this paper, the engineering background is that during the SAGD process at a Canadian oil sands block, trying to obtain the relevant evaluation and prediction about the steam chamber. Multi-physics flexibility and computational performance of COMSOL gave us the idea to model SAGD one of the popular thermal method of oil recovery. This article focuses on the model framework and the simulation results about the numerical software Comsol in numerical simulation of SAGD process.
\end{abstract}

\section{Introduction}

Conventional hydrocarbon resources become scarcer every day, while world energy needs continue to increase. For this reason, energy producing companies increasingly exploit nonconventional hydrocarbon resources such as heavy oil and bitumen [1]. This article based on SAGD project cases happened under such a big trend. During the SAGD process, there will involves the thermal dynamic related to multiphase flow and thermal coupling between pore pressure and thermal expansion. As a result, we chose the Comsol Multiphysics software as the tool of numerical simulation. The numerical simulation research in this paper was under the support of the production data and indoor experimental data. The core of the indoor experiment is from Canada's oil sands blocks in order to ensure the reference value about the experimental data. We got a relatively accurate simulation and reduction about the geomechanics conditions in production block based on the numerical simulation of Comsol. At the same time, we gave rock mechanics parameters of the geometric model via the existing geological data. Specific simulation will show through the following four parts.

\section{Physical Background}

In the whole process of heavy oil thermal recovery, mainly considering gravity, thermal expansion, pore pressure, as well as the various geological factors such as horizontal geostress combination. For the geological structure, the formation above reservoir is separated layer and the upper cover, as shown in figure 1. Because of the hot steam main emissions to the reservoir, and the reservoir itself is porous media, so a combination of various physical fields mainly occurs in the reservoir, at the same time the temperature field and stress field of the isolation layer and the covering layer will change along with the change of reservoir [2]. First of all, hot steam injection directly led to the change of the temperature field, then the change of thermal stress field caused by temperature field change. On the other hand, the change of temperature field will pass up, from the reservoir to the interlayer and the upper layer, the thermal expansion caused by temperature transfer will lead to thermal stress changes between the separated layer and the upper cover. Due to the reservoir is a porous media, with the heavy oil thermal recovery process, pore pressure is constantly changing, the change of pore pressure field and the change of the thermal stress field coupling each other, leading to the geological mechanics properties change of oil sands block. Mastery of this change is directly related to production safety, and this is what we want to get by Comsol simulation. 


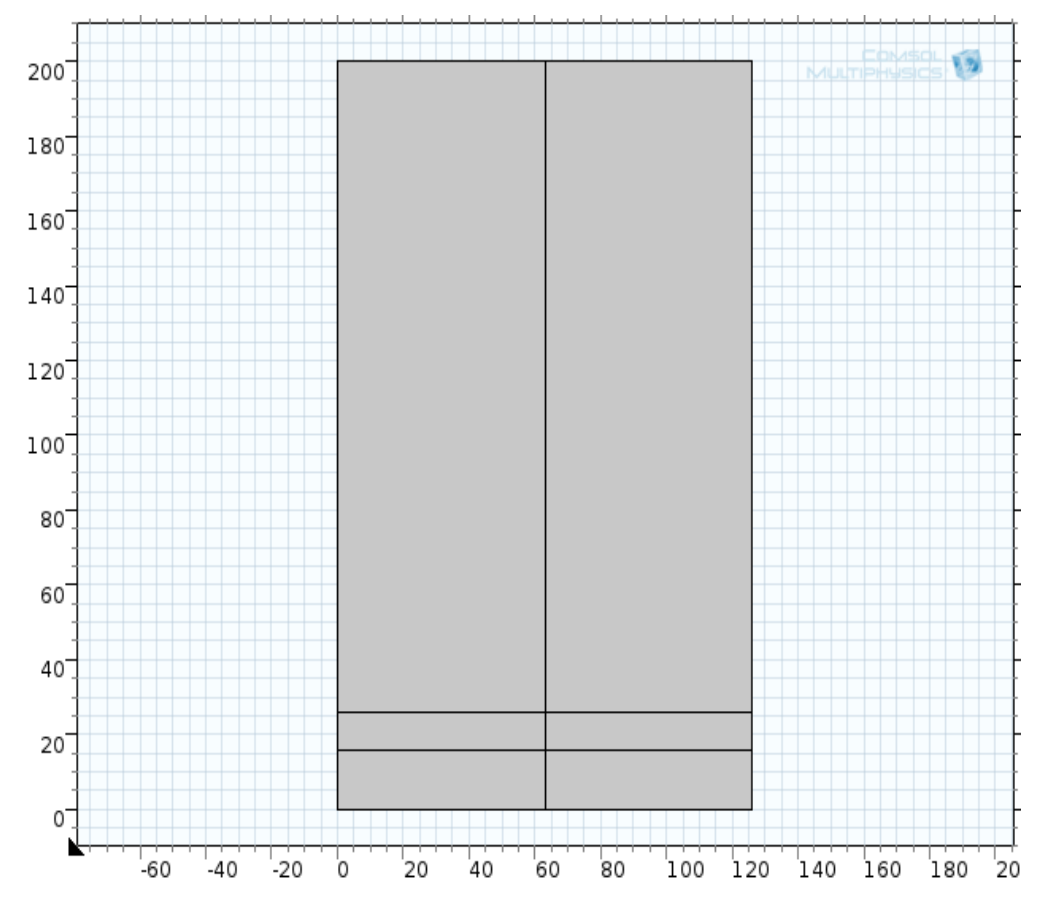

Fig. 1 SAGD Geometric Model

\section{Simulation Logic}

Use two Solid Mechanics fields and a Heat Transfer field to complete the simulation of the whole process of SAGD [3]. First of all, get through the field of Solid Mechanics 1 to gain the ground stress field distribution, which was under initial boundary conditions such as gravity and horizontal ground stress. Then, pass the stress field obtained in the field of Solid Mechanics 1 to the field of Solid Mechanics 2 as the initial stress field of Solid Mechanics 2. Be careful that only pass the stress field, deformation does not pass, initial deformation of Solid Mechanics 2 is zero. Applied the Solid Mechanics 1 and Solid Mechanics 2 field in the same boundary conditions, at the same time, have to realize the simulation of thermal expansion, pore pressure and plastic in the Solid Mechanics 2 .What particularly needs to pay attention to is that, the temperature field of the thermal expansion module must be the temperature field after delivery. What we had known was only the temperature field of reservoir, so we have to get the temperature field of the isolation layer and the upper cover, and it must be done by Heat Transfer physical fields of the Comsol software, namely realizing the delivery of temperature field from the reservoir to the isolation layer and the covering layer. Finally finish the mesh of the whole geometric model.

\section{Geometric Model and Parameters}

By engineering practice, combined with the actual geological conditions, we establish a geometric model shown in figure 1, from bottom to top were reservoir and interlayer respectively and the upper layer, and the thickness of the three layers were 16 meters, 10 meters, 174 meters. On the other hand, the formation of rock mechanics parameters are obtained by experiment, we obtained from the various strata in the process of oilfield exploitation of full diameter core indoor rock mechanics parameters of the comprehensive experiment was carried out, including ordinary triaxial compression experiment, acoustic experiment, acoustic emission experiment, and then get a reservoir rock mechanics parameters of rock, such as elastic modulus, poisson's ratio, cohesion, internal friction angle, etc.

\section{The Initial Conditions and Boundary Conditions}

The Solid Mechanics 2 is the key to numerical simulation, the boundary conditions of the the Solid Mechanics 2 are the same as the Solid Mechanics 1, including gravity, ground stress and roll shaft support, while the initial conditions of solid mechanics field 2, through inheritance to get the stress distribution in 
the field of Solid Mechanics 1.On the other hand, use the linear elastic module of Solid Mechanics 2 to realize the coupling of plastic, thermal expansion and pore pressure.

\section{Conclusion}

The Mises stress field after coupling (see Fig.2)

COMSOL model of the SAGD process has been successfully built and tested [4]. Although computational performance of the model is not still competitive at the moment in comparison to the industrial reservoir simulator, the multi-physics flexibility and diversity of COMSOL based applications offer the promising possibilities in research on novel methods of oil recovery.

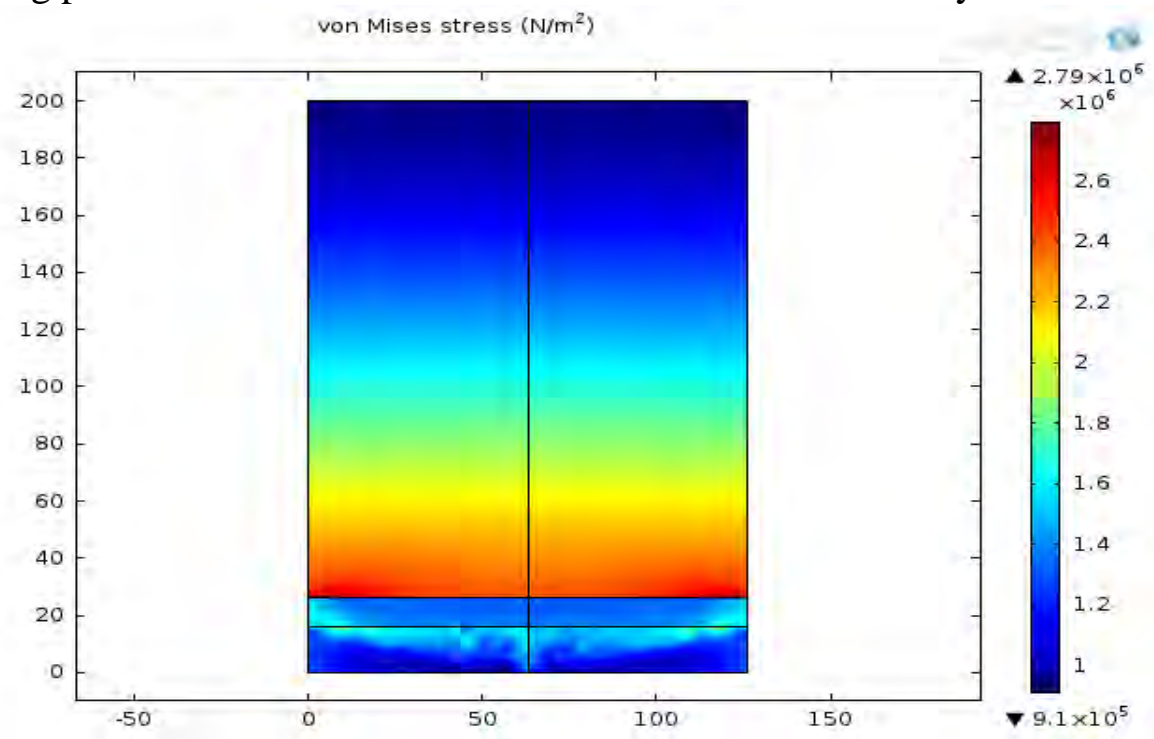

Fig. 2 the Mises Stress Field After Coupling

\section{References}

[1]Butler R.M, Steam-assisted gravity drainage:Concept, Development, Performance and Future, Journal of Canadian Petroleum Technology,Volume 33, No 2, 44-50 (1994).

[2] van Genuchten M.Th. A closed-form equation for predicting the hydraulic conductivity of unsaturated soils. Soil Sci. Am. J., Volume 44,892-898 (1980).

[3] Computer Modelling Group Ltd., STARS Manual, Calgary, Alberta, October 2004.

[4] Information on http://www.cn.comsol.com. 